\title{
Diversity of Insect and Non Insect Pest Infesting Karanja, Pongamia pinnata (L.) Pierre at Bhubaneswar, Odisha, India and their Natural Enemies
}

\author{
Jyoti Kumari $^{1 *}$, M. K. Tripathy ${ }^{2}$ and Harekrushna Das ${ }^{1}$ \\ ${ }^{1}$ Department of Natural Resource Management, College of Forestry, \\ OUAT, Bhubaneswar, India \\ ${ }^{2}$ Department of Entomology, CA, OUAT, Bhubaneswar -3, India \\ *Corresponding author
}

\section{A B S T R A C T}

\section{Keywords}

Leaf minor, Stem galls, Population dynamics, and Eriophyid mites

\section{Article Info}

Accepted:

14 June 2020

Available Online:

10 July 2020
In an study on diversity of insect and non-insect pests infesting karanja, Pongamia pinnata (1.) pierre and their natural enemies at, a total of 28 species of insects including an acarine and 12 species of natural enemies were recorded during 2018-2019. The phytophagus insects were categorized under 5 groups viz. Foliage feeders or defoliators, Stem borers ,Bark borers ,sap feeders and seed feeders Peak infestation due to eriophyid mite was recorded during second fortnight of May 2018 (50.35\%).and maximum number of galls per branch due to agromyzid fly infestation was observed in $1^{\text {st }}$ fortnight of August $(6.45$ galls per branch) and maximum mean number of maggots recovered per gall was 8.50 in $1^{\text {st }}$ fortnight of August2018. The mean percentage plant infestation due to leaf miner attack was varied from minimum of $15 \%$ infestation during $1^{\text {st }}$ fortnight of December 2018 to a maximum of $89.00 \%$ as recorded during first fortnight of October, 2018.

\section{Introduction}

Pongamia pinnata (L.) Pierre is most commonly grown in humid and sub tropic environment, most commonly in coastal areas with its roots in freshwater or salt water but best growth is found in deep well drained sandy loam soil with assured moisture content and can also grow on sandy soils but do not grow well on dry sands (Orwa et al., 2009). It is also used in herbal medicine which is known as botanical medicine or as phytotherapy or phytomedicine which means a plant or plant part is used to make medicine to assist in the healing process during illness and disease. The roots of Pongamia pinnata (L.) Pierre is good for cleaning foul ulcers, cleaning teeth, strengthening gums and gonorrhea. Among various constraints causing losses in large trees, insects are the major one, which deteriorates the quality and the quantity of trees, shrubs, herbs as well as their produce There are about 30 species of insect pests recorded to cause damage to Pongamia pinnata (L.) Pierre rose usually as avenue and strip plantations on marginal lands. They include leaf miner defoliator, shoot borer, sap suckers, flower feeder etc. 
(Orwa et al., 2009; Sundararaj et al., 2005) reported a checklist of insects infesting Pongamia pinnata (L.) Pierre in India. This includes about 64 species of insects representing 21 families under six orders and one species of mite belonging to the Family Eriophyid of the Order Acarina. Keeping in view of the immediate need for understanding the arthropods diversity and population dynamics of important pests associated with Pongamia pinnata (L.) Pierre and their relationship with weather parameters, investigation was undertaken during the 20182019 in Bhubaneswar, Odisha with the objectives to study the diversity of different arthropods associated with Pongamia pinnata (L.) Pierre at Bhubaneswar location and To study the population dynamics of major foliage feeding insect viz. Leaf miner and some important leaf feeders associated with Pongamia pinnata (L.) Pierre.

\section{Materials and Methods}

The details of materials and methodology used in these experiments are discussed here under.

\section{Location of the experimental site and} season

The present investigation" was carried out during May 2018 to April 2019 at different locations viz., Silviculuture Research Station, Ghatikia, Bhubaneswar, and Seedling nursery of College of Forestry located in OUAT campus, Bhubaneswar, situated at an elevation of $25.9 \mathrm{~m}$ above MSL at $20^{\circ} 15^{\prime} \mathrm{N}$ latitude and $85^{\circ} 52^{\prime}$ E longitude. It is situated in the East \& South East Coastal Plain zone of Odisha, which falls under tropical wet-dry or tropical Savanna forest type. The general climate is warm and humid. Highest rainfall of $140 \mathrm{~mm}$ was recorded during August I fortnight whereas the total rainfall recoded from October second fortnight to April first fortnight was $3.88 \mathrm{~mm}$. Afternoon relative humidity was more than $70 \%$ for the period from June second fortnights to September second fortnight.

\section{Identification of insect pests and their natural enemies}

Flying and slow moving insects were collected by means of a swift net and preserved appropriately. Immature stages of Lepidopterans and coleopterons insects were reared in the laboratory up to adult stage in natural food medium in plastic cups of 4-inch diameter and 4-inch depth after following all the sanitation practices. Identification was established either by personnel experience or from entomology museum or by consulting professors of entomology Department or from Internet sources. (Patil et al., 2016, Tripathy et al., 2018)Insect predators and parasites were identified either by directly collected from field or by rearing of field collected countable number of eggs or keeping the blotched leaves along with water soaked cotton wrapped in leaf base Photographs were taken by Stereoscopic binocular microscope modelCDGT510CCD) in the post graduate laboratory fitted with computer and provided with specific software. More critical detail of minute powder post beetles setae types of collected insect's cloaca apertures and their measurements was obtained with scanning electron microscope located in Central laboratory, OUAT, Bhubaneswar.

\section{Population dynamics of major foliage feeding insect's viz. leaf miner and some important leaf feeders}

Abundance of defoliating pests of Karanj was recorded at two different locations viz., Silviculuture Research Station, Bhubaneswar, College of Forestry, OUAT campus, Bhubaneswar. At each location, plants of each category as detailed below with uniform 
growth pattern and age were selected (I) Seedlings- 0-1-year-old (ii) Saplings- 1-3year-old (iii)Plants- more than 3 years old. For seedlings and plants of 1-3 years old, all the leaves are selected from 30 plants and sampled. For more than 3 years old plant, five terminal twigs were randomly selected from the lower and middle canopy of each plant and 10 plants were taken, from which leaves from each twig were sampled for observing number of healthy and damaged leaves.

Population densities of different categories of pests are expressed as per Rout (2017) and standard error in each case has been determined by putting the standard formula. Thus percent infestation due to defoliation was worked out by using standard formula,

$\%$ defoliation $=$ Number of infested leaves $\times 100$ Number of leaves examined

\section{Results and Discussion}

From Table- 01 and 02 it is evident that 28 species of insects and non-insect pests and 12 species of predators were recorded from Pongamia pinnata (L.) Pierre at Bhubaneswar during the period of study (2018-2019). Taxonomically, 5 species of Coleoptera, 9 species of Lepidoptera, 8 species of Hemiptera, 1 species of Acarina, 4 species of Orthoptera and 1 species each from Diptera and Isoptera were recorded. A total of 12 species of natural enemies have been recorded out of which 5 species are spiders, 3 species from Hymenoptera and 1 species each from Odonata, Neuropteran, Mantodea and Hemiptera. While categorising the insects as per the nature of damage out of total species recorded at Bhubaneswar situation, 53.84\% constitute the foliage feeders, $32.14 \%$ constitutes the sap feeders, 7. 69\% are pod borers, $7.69 \%$ are bark feeders and $3.84 \%$ are stem borers only. A brief description of the insect pests attacking the plants along with nature of damage as well as their natural enemies is given below.

\section{Foliage feeders}

\section{Pongamia leaf miner- (Lithocolletis virgulata Meyrick)}

The mining was done by the tiny caterpillar which results in formation of white circular blotches on the leaves. In severe damage all the leaves are attacked. Female lay eggs on dorsal side of fresh leaves which hatch in about a week time and starts feeding on chlorophyll of the leaves. Initially they mine in irregular fashion. This give the shape of blotch to the mined portion, the larva feeds about a fortnight and pupate within the blotch the adult of which is a small moth about $2 \mathrm{~mm}$ long silvery grey, emerges by making a small emergence hole in the upper side to start a new life.

\section{Common banded awl (Hasora chromus Cramer)}

The larvae of Hasora chromus Cramer was observed as a major defoliator of $P$. pinnata (L.) Pierre mostly during the rainy season. Larvae folds the leaves and feeds within it, particularly on young and tender leaves. The head was large, black with four white stripes on the dorsal side towards the prothorax up to last abdominal segment of the body. Adult butterflies are brownish in colour with prominent eyes and short antennae.

\section{Bagworm- (Pteroma plagiophleps Hampson)}

Bagworm infestation was recorded during the Rabi season. They are mostly attached to the stem and old leaves and pupa are mostly hanging from the leaves. Sexual dimorphism is well pronounced in adult of both the sexes. Males have well developed wings, legs and mouthparts whereas adult female is devoid of well-developed wings, legs and mouthparts. 
Hairy caterpillar- (Euproctis sp.) Causes defoliation of host plants. Early instar larvae of hairy caterpillar, Euproctis sp. was observed feeding on the green part of the leaves and skeletonized them but later instar larvae feed completely. The soft apical shoots of plants are also damaged at times.

Indian sunbeam- (Curetis thetis Drury) Caterpillar they feeds on the leaves and feeding symptoms result in the formation of holes on the leaves. These are mostly seen in the Kharif season.

Common cerulean- (Jamide sceleno Cramer) the forewings have the terminal margin narrowly edged with black that broadens very slightly towards the apex of wings. Larvae are found feeding on the leaves of Pongamia pinnnata (L.) Pierre.

Karanj defoliator (Maruca ambionalis Felder) was recorded as the another important defoliator of Pongamia pinnata (L.) Pierre during the post rainy period at Bhubaneswar. The larvae caused webbing in young leaves and remained inside the webs and fed on the leaf tissue.

Leaf feeding beetle (Diabrotica sp.) Small to medium sized beetles, slender, active in habitat and mostly found during summer months. Both adults and grubs of the leaf beetle were observed feeding on the leaves by making holes. The elytra did not cover the abdomen fully.

Leaf gall mite- (Aceria pongamiaeKeifer) Belongs to Eriophyidae of Acarina. Eriophyid mite induces finger like or pouched galls on both surfaces of the infested leaf. Most of infestation of Aceria pongamia Keifer was observed during the post rainy season during the month of May - October2018.Body cylindrical, whitish or creamy coloured, feather claw 7 rayed, shield design narrowly truncated in front, median line not clear; a median line close to one another, complete meet at rear shield. Abdomen with 70 rings uniformly microtuberculate.

Stem gall fly- Maggots are seen feeding in the soft tender twigs resulting in swellings and galls on the stem. Maggots are whitish apodous having size of 3-4 $\mathrm{mm}$ in length whereas adult fly is black in colour and have size of about 3-4 mm

Grasshopper -Found feeding on theyoung seedlings a total of 3 species was recorded.

Tree hopper -Both nymphs and adults of tree hopper were found sucking sap from tender leaves and shoots of $P$. pinnata (L.) Pierre.

Unidentified weevil- Both grub and adult are seen feeding on $P$. pinnata (L.) Pierre leaves leading to small circular holes on the tender leaves. Grubs are black in colour with 3-4 mm in size and having well developed snout.

Stem borer- (Leiopus nebulosus Linnaeus) Both grub and adult are seen feeding on Pongamia stems by boring holes on the stem. Adult are $3-4 \mathrm{~cm}$ long and $1-1.5 \mathrm{~cm}$ wide with long serrated have long antenna. Adult are greyish in colour with black coloured patches on the elytra (Fig.-)

Bark borer - (Indarbela quadrinotata Walker) Bark borer was observed infesting the main stem of the P. pinnata (L.) Pierre. Infested stem could be identified by the holes and webbing of frass material along with excreta of the larvae feeding inside.

\section{Sap feeders}

Mealybugs (Ferissia virgate Cockerell)Mealy bugs caused damage by sucking the sap of the leaves and young shoots of $P$. pinnata (L.) Pierre. Feeding continuously retarded growth as a result yellowing, withering and falling of leaves were noticed. 
Bug (Homoeocerus biguttata Westwood)

Both nymphs and adults sucked the sap of young leaves. Body of the bugs was green in colour with brown coloured wing. Adult bug measured about 12.0 to $15.0 \mathrm{~mm}$ in length and 3.0-4.0 $\mathrm{mm}$ in width.

Stink bug (Nezara viridula Linnaeus) Both nymph and adults green bug, Nezara virudula were observed as sucking pest on young shoots and leaves of Pongamia. Major appearance was observed during post rainy and winter months.

Red cotton bugs (Dysdercus cingulatus Fabricius) Both nymphs and adults of red cotton bugs, found sucking sap from tender leaves, petioles and shoots in early stages and then infest flower buds of $P$. pinnata (L.) Pierre.

\section{Pod borer}

Pulse beetle (Callosobruchus sp.) Grubs are seen inside the pods, cream coloured, scarabaei form, fleshy and feeds inside the seeds

Pod borer (Ephestia sp.)-The larvae entered inside the pods at an early stage of larval instar such that their entry hole got healed up later due to which it was difficult to distinguish an infested fruit from an uninfected one by visual observation. Infestation of pod borer was observed to be serious on Pongamia pods where seeds were fallen on dampy places.

\section{Natural enemies associated with pests of} Pongamia pinnata (L.) Pierre

Red ant -Red ant, were observed associated with infesting leaves of $P$. pinnata(L.) Pierre by making nests by joining on an average 4-5 leaves. The ants lived within the nests and came out at the slightest disturbance of the trees.

Black ant - Generally black ants congregating near honeydews.

Green lace wing -Chrysoperla sp. was observed as a predator on soft bodied insects such as mealy bugss and plant hoppers

Mantids: Mantid was observed predating on flying insects which included various species such as plant hoppers beetles, moths and butterflies

Spiders: Various species of spiders were recorded on Pongamia sp. during the present studies. It was recorded to be a polyphagous predator of various species of insects.

Predatory stink bug- Stink bug predates on the larval stages of various insects attacking Pongamia pinnata (L.) Pierre.

Data in Table. 3 revealed the mean percentage defoliation/ plant. Peak defoliation was recorded after the onset of monsoon from second fortnight of July, 2018 to second fortnight of October, 2018. No leaf infestation due to the mite Aceria pongamia Keifer was recorded during the months of December to February second fortnight The initiation of infestation could be coupled with summer when leaf sprouting occurs during early days of March. Peak infestation was recorded during second fortnight of May 2018 $(50.35 \%)$. Mean number of gall per pinna also follow similar trends with lowest number of galls per pinna was $5.00 \%$ as recorded during $1^{\text {st }}$ fortnight of December to 29 galls per pinna in $1^{\text {st }}$ fortnight of May, 2018. Mean percentage branch infestation in each plant due to agromyzid varied from nil $\left(2^{\text {nd }}\right.$ fortnight of May, 2018 to $1^{\text {st }}$ fortnight of January, 2019) to a maximum of $43.00 \%$ ( $1^{\text {st }}$ fortnight of August,2018) Maximum number of galls per branch was observed in $1^{\text {st }}$ fortnight of August (6.45 galls per branch) and maximum mean number of maggots recovered per gall was 8.50 in $1^{\text {st }}$ fortnight of August. 
Table.1 Diversity of insect and non-insect pest infesting karanja Pongamia pinnata (L.) Pierre at Bhubaneswar, Odisha, India

\begin{tabular}{|c|c|c|c|c|c|c|}
\hline $\begin{array}{l}\text { Sl. } \\
\text { no. }\end{array}$ & $\begin{array}{c}\text { Common } \\
\text { Name }\end{array}$ & Scientific Name & Family & Order & Status & $\begin{array}{c}\text { Infested } \\
\text { plant parts }\end{array}$ \\
\hline \multicolumn{7}{|c|}{ A) Foliage feeders } \\
\hline 1 & $\begin{array}{l}\text { Pongamia } \\
\text { leaf miner }\end{array}$ & Lithocolletis virgulataMeyrick & Gracillaridae & Lepidoptera & Major & Foliage \\
\hline 2 & $\begin{array}{c}\text { Common } \\
\text { banded awl }\end{array}$ & Hasora chromusCramer & Hesperidae & Lepidoptera & Major & Foliage \\
\hline 3 & Bagworm & Pteroma plagiophlepsHampson & Psychiidae & Lepidoptera & Major & Foliage \\
\hline 4 & $\begin{array}{c}\text { Hairy } \\
\text { catterpillar }\end{array}$ & Euproctissp.Hubner & Lymantriidae & Lepidoptera & Minor & Foliage \\
\hline 5 & $\begin{array}{c}\text { Indian } \\
\text { sunbeam }\end{array}$ & Curetis thetisDrury & Lycaenidae & Lepidoptera & Minor & Foliage \\
\hline 6. & $\begin{array}{l}\text { Common } \\
\text { cerulean }\end{array}$ & Jamides celenoCramer & Lycaenidae & Lepidoptera & Minor & Foliage \\
\hline 7. & $\begin{array}{c}\text { Karanj } \\
\text { defoliator }\end{array}$ & Maruca ambionalisFelder & Crambidae & Lepidoptera & Major & Foliage \\
\hline 8. & $\begin{array}{l}\text { Leaf gall } \\
\text { mite }\end{array}$ & Aceria pongamiaeKeifer & Eriophyidae & Acarina & Major & Leaf \\
\hline 9. & $\begin{array}{l}\text { Stem gall } \\
\text { making fly }\end{array}$ & Unidetified & Agromyzidae & Diptera & Major & Top shoots \\
\hline 10. & $\begin{array}{c}\text { Brown } \\
\text { Grasshopper }\end{array}$ & Diabolocatantops axillarisJago & Acrididae & Orthoptera & $\begin{array}{l}\text { Minor in } \\
\text { seedlings }\end{array}$ & $\begin{array}{c}\text { Foliage of } \\
\text { seedligs }\end{array}$ \\
\hline 11. & $\begin{array}{l}\text { Long horned } \\
\text { grasshopper }\end{array}$ & Ducetia japonica Thunberg & Tettigoniidae & Orthoptera & $\begin{array}{l}\text { Major in } \\
\text { seedlings }\end{array}$ & $\begin{array}{c}\text { Foliage of } \\
\text { seedlings }\end{array}$ \\
\hline 12. & Katydid & Pterophylla camellifoliaFabricius & Tettigoniidae & Orthoptera & $\begin{array}{l}\text { Minor } \\
\text { in } \\
\text { seedlings }\end{array}$ & Foliage \\
\hline 13. & $\begin{array}{c}\text { Short horned } \\
\text { grasshopper } \\
\text { Weevil }\end{array}$ & Oxya hylaServille & Acrididae & Orthoptera & $\begin{array}{l}\text { Minor in } \\
\text { seedlings }\end{array}$ & Seedlings \\
\hline 14. & $\begin{array}{c}\text { Leaf feeding } \\
\text { beetle }\end{array}$ & Diabrotica sp. & Chrysomelidae & Coleoptera & Minor & Leaf \\
\hline 15 & *Weevil & unidentified & Curculionidae & Coleoptera & Major & $\begin{array}{c}\text { Feed on } \\
\text { tender }\end{array}$ \\
\hline
\end{tabular}




\begin{tabular}{|c|c|c|c|c|c|c|}
\hline & & & & & & $\begin{array}{c}\text { leaves } \\
\text { making } \\
\text { small holes }\end{array}$ \\
\hline \multicolumn{7}{|c|}{ B) STEM BORER } \\
\hline 16. & $\begin{array}{l}\text { Longicorn } \\
\text { beetle }\end{array}$ & Leiopus nebulosus Linnaeus & Cerambycidae & Coleoptera & Borers & Stem \\
\hline \multicolumn{7}{|c|}{ C) BARK BORER } \\
\hline 17 & Bark borer & Indarbela quadrinotata Walker & Arbellidae & Lepidoptera & Minor & $\begin{array}{l}\text { Bore the } \\
\text { bark }\end{array}$ \\
\hline 18 & Termite & Odontotermes obesus Rambur & Termitidae & Isoptera & Major & $\begin{array}{c}\text { Feeds on } \\
\text { bark and } \\
\text { roots of } \\
\text { fresh } \\
\text { transplants. }\end{array}$ \\
\hline \multicolumn{7}{|c|}{ D) SAP FEEDER } \\
\hline 19 & Mealybug & Nipaecoccus viridis Newstead & Pseudococcidae & Hemiptera & Major & $\begin{array}{c}\text { Foliage, } \\
\text { twigs }\end{array}$ \\
\hline 20 & $\begin{array}{l}\text { Two tailed } \\
\text { mealy bug }\end{array}$ & Ferrisia virgata Cockerell & Pseudococcidae & Hemiptera & Major & Foliage \\
\hline 21 & Bug & Homoeocerus biguttatus Westwood & Coreidae & Hemiptera & Major & Foliage \\
\hline 22 & $\begin{array}{l}\text { Red cotton } \\
\text { bug }\end{array}$ & $\begin{array}{l}\text { Dysdercus cingulatus } \\
\text { Fabricius }\end{array}$ & Pyrrhocoridae & Hemiptera & Minor & Foliage \\
\hline 23 & $\begin{array}{l}\text { Metallic } \\
\text { shield bug }\end{array}$ & Chrysochoris stolli Wolf & Scutelleridae & Hemiptera & Minor & Foliage \\
\hline 24 & $\begin{array}{l}\text { Spotted } \\
\text { lanternfly }\end{array}$ & Lycorma delicatula White & Fulgoridae & Hemiptera & Minor & Foliage \\
\hline 25 & Tree hopper & unidentified & Membracidae & Hemiptera & Minor & Foliage \\
\hline 26 & Bug & unidentified & - & Hemiptera & Minor & Foliage \\
\hline \multicolumn{7}{|c|}{ E) SEED FEEDERS } \\
\hline 27 & Pulse beetle & Callosobruchus sp. & Bruchidae & Coleoptera & $\begin{array}{l}\text { Minor } \\
\text { and feeds } \\
\text { on side } \\
\text { seeds }\end{array}$ & Pod borer \\
\hline 28 & Pod borer & Ephestia sp. & Pyralidae & Lepidoptera & $\begin{array}{c}\text { Minor but } \\
\text { seed } \\
\text { feeder }\end{array}$ & Pod borer \\
\hline
\end{tabular}


Table.2 Succession of natural enemies of major insect pests infesting Pongamiapinnata (L.)

Pierre at Bhubaneswar (2018-2019)

\begin{tabular}{|c|c|c|c|c|c|c|}
\hline $\begin{array}{l}\text { Sr. } \\
\text { no. }\end{array}$ & $\begin{array}{c}\text { Common } \\
\text { name }\end{array}$ & Scientific name & Family & Order & Type & Remarks \\
\hline 1. & $\begin{array}{l}\text { Emerald- } \\
\text { banded } \\
\text { skimmer }\end{array}$ & Cratilla lineata & Anisoptera & Odonata & Predator & $\begin{array}{c}\text { Naids are } \\
\text { predators in } \\
\text { swampy } \\
\text { areas }\end{array}$ \\
\hline 2. & $\begin{array}{c}\text { Two } \\
\text { striped } \\
\text { jumping } \\
\text { spider }\end{array}$ & $\begin{array}{l}\text { Telamonia dimidiate } \\
\text { Simon }\end{array}$ & Salticidae & Araneae & Predator & $\begin{array}{l}\text { Feeds on } \\
\text { small } \\
\text { insects }\end{array}$ \\
\hline 3. & $\begin{array}{c}\text { Heavy } \\
\text { bodied } \\
\text { jumping } \\
\text { spider }\end{array}$ & $\begin{array}{l}\text { Hyllus semicupreus } \\
\text { Simon }\end{array}$ & Salticidae & Araneae & Predator & $\begin{array}{l}\text { Feeds on } \\
\text { small } \\
\text { insects }\end{array}$ \\
\hline 4. & Spider & Neoscona sp. & Araneidae & Araneae & Predator & $\begin{array}{c}\text { Feeds on } \\
\text { small } \\
\text { insects }\end{array}$ \\
\hline 5. & $\begin{array}{l}\text { Hasselt's } \\
\text { spiny } \\
\text { spider }\end{array}$ & $\begin{array}{l}\text { Gaster acanthahasselti } \\
\text { Koch }\end{array}$ & Araneidae & Araneae & Predator & $\begin{array}{l}\text { Feeds on } \\
\text { small } \\
\text { insects }\end{array}$ \\
\hline 6. & $\begin{array}{l}\text { Giant } \\
\text { golden orb- } \\
\text { weaver }\end{array}$ & Nephilla Leach & Araneidae & Araneae & Predator & $\begin{array}{c}\text { Feeds on } \\
\text { small } \\
\text { insects }\end{array}$ \\
\hline 7. & Red ant & Solenopsis Westwood. & Formicidae & Hymenoptera & Visiting & $\begin{array}{c}\text { Seen on } \\
\text { honeydew }\end{array}$ \\
\hline 8. & Black ant & Lasiusniger Linneaus & Formicidae & Hymenoptera & Visiting & $\begin{array}{l}\text { Seen on } \\
\text { honeydew }\end{array}$ \\
\hline 9 & $\begin{array}{l}\text { Leaf miner } \\
\text { parasitoid }\end{array}$ & unidentified & Eulophidae & Hymenoptera & Parasioid & $\begin{array}{l}\text { Found } \\
\text { inside } \\
\text { mines }\end{array}$ \\
\hline 10 & $\begin{array}{c}\text { Green } \\
\text { lacewing }\end{array}$ & Chrysoperla sp. & Chrysopidae & Neuroptera & Predator & $\begin{array}{l}\text { Found in } \\
\text { plants }\end{array}$ \\
\hline 11 & $\begin{array}{l}\text { Preying } \\
\text { mantid }\end{array}$ & Mantis sp. & Mantidae & Mantodea & Predator & $\begin{array}{l}\text { Found in } \\
\text { plants }\end{array}$ \\
\hline 12 & $\begin{array}{l}\text { Green stink } \\
\text { bug }\end{array}$ & Nezara viridula Linnaeus & Pentatomidae & Hemiptera & Predator & $\begin{array}{l}\text { Found in } \\
\text { plants }\end{array}$ \\
\hline
\end{tabular}

(*)- unidentified 
Table.3 Infestation of different gall forming insects in Pongamia pinnata (L.) Pierre (2018-2019) at Bhubaneswar

\begin{tabular}{|c|c|c|c|c|c|c|c|c|c|c|}
\hline \multirow{2}{*}{$\begin{array}{l}\text { Period of } \\
\text { observation }\end{array}$} & \multirow{2}{*}{$\begin{array}{l}\text { Mean (\%) } \\
\text { defoliation } \\
\text { per plant }\end{array}$} & \multicolumn{5}{|c|}{ Infestation due to mite (AceriapongamiaeKeifer) } & \multicolumn{4}{|c|}{ Infestation due to Agromyzid } \\
\hline & & $\begin{array}{c}\text { Mean plant } \\
\text { infestation } \\
\%\end{array}$ & $\begin{array}{c}\text { Mean } \\
\text { percentage } \\
\text { compound } \\
\text { leaf } \\
\text { infestation }\end{array}$ & $\begin{array}{l}\text { Mean gall } \\
\text { intensity of } \\
\text { infested } \\
\text { compound } \\
\text { leaves }\end{array}$ & $\begin{array}{l}\text { Mean } \\
\text { percentage } \\
\text { simple leaf } \\
\text { infestation }\end{array}$ & $\begin{array}{l}\text { Mean no. } \\
\text { of galls per } \\
\text { pinna }\end{array}$ & $\begin{array}{c}\text { Mean \% } \\
\text { plant } \\
\text { infestation }\end{array}$ & $\begin{array}{c}\text { Mean \% } \\
\text { branch } \\
\text { infestation }\end{array}$ & $\begin{array}{l}\text { Mean gall } \\
\text { intensity } \\
\text { per branch }\end{array}$ & $\begin{array}{c}\text { Mean } \\
\text { number of } \\
\text { maggot } \\
\text { recovered } \\
\text { per gal }\end{array}$ \\
\hline MAY I, 2018 & $\begin{array}{l}12.10 \pm 0.05 \\
(11.00-12.75)\end{array}$ & $\begin{array}{c}38.20 \pm 1.05 \\
(35.00-45.00)\end{array}$ & $\begin{array}{c}45.45 \pm 4.50 \\
(30.00- \\
50.00)\end{array}$ & $\begin{array}{c}42.25 \pm 2.50 \\
(35.00- \\
60.00)\end{array}$ & $\begin{array}{c}26.00 \pm 2.50 \\
(23.00- \\
35.00)\end{array}$ & $\begin{array}{c}29.00 \pm 1.50 \\
(22.00- \\
32.00)\end{array}$ & $\begin{array}{c}15.00 \pm 0.05 \\
(10.00- \\
20.00)\end{array}$ & $\begin{array}{c}30.00 \pm 0.55 \\
(25.00- \\
35.00)\end{array}$ & $\begin{array}{c}4.35 \pm 1.05 \\
(2.00-6.00)\end{array}$ & $\begin{array}{c}6.00 \pm 0.05 \\
(2.00-11.00)\end{array}$ \\
\hline $\begin{array}{l}\text { MAY II } \\
2018\end{array}$ & $\begin{array}{l}9.64 \pm 0.75 \\
(8.50-10.00)\end{array}$ & $\begin{array}{c}50.35 \pm 3.08 \\
(50.00-55.00)\end{array}$ & $\begin{array}{c}46.75 \pm 3.50 \\
(30.00- \\
50.00)\end{array}$ & $\begin{array}{c}45.75 \pm 1.50 \\
(40.00- \\
50.00)\end{array}$ & $\begin{array}{c}27.75 \pm 2.50 \\
(23.00- \\
32.00)\end{array}$ & $\begin{array}{c}28.25 \pm 2.50 \\
(22.00- \\
33.00)\end{array}$ & $\begin{array}{c}18.00 .15 \pm 0 \\
01 \\
(10.00- \\
25.00)\end{array}$ & $\begin{array}{c}31.33 \pm 0.60 \\
(25.00- \\
35.00)\end{array}$ & $\begin{array}{c}4.15 \pm 2.01 \\
(3.00-8.00)\end{array}$ & $\begin{array}{c}6.50 \pm 0.05 \\
(3.00-8.00)\end{array}$ \\
\hline JUNE I 2018 & $\begin{array}{l}13.71 \pm 0.82 \\
(13.0-14.00)\end{array}$ & $\begin{array}{c}28.00 \pm 2.05 \\
(25.00-35.00)\end{array}$ & $\begin{array}{c}37.33 \pm 2.05 \\
(20.00- \\
38.00)\end{array}$ & $\begin{array}{c}40.20 \pm 1.75 \\
(35.00- \\
50.00)\end{array}$ & $\begin{array}{c}25.45 \pm 2.50 \\
(13.00- \\
32.00)\end{array}$ & $\begin{array}{c}25.45 \pm 3.50 \\
(20.00- \\
35.00)\end{array}$ & $\begin{array}{c}20.05 \pm 0.03 \\
(15.00- \\
25.00)\end{array}$ & $\begin{array}{c}34.50 \pm 0.55 \\
(30.00- \\
40.00)\end{array}$ & $\begin{array}{c}4.45 \pm 2.03 \\
(3.00-7.50)\end{array}$ & $\begin{array}{c}7.5 \pm 0.05 \\
(3.00-12.00)\end{array}$ \\
\hline $\begin{array}{l}\text { JUNE II } \\
2018\end{array}$ & $\begin{array}{l}16.80 \pm 0.09 \\
(15.85-17.05)\end{array}$ & $\begin{array}{c}36.90 \pm 2.06 \\
(35.00-45.00)\end{array}$ & $\begin{array}{c}39.40 \pm 4.50 \\
(20.00- \\
35.00)\end{array}$ & $\begin{array}{c}38.75 \pm 2.50 \\
(20.00- \\
45.00)\end{array}$ & $\begin{array}{c}22.45 \pm 2.50 \\
(13.00- \\
25.00)\end{array}$ & $\begin{array}{c}20.45 \pm 1.50 \\
(12.00- \\
25.00)\end{array}$ & $\begin{array}{c}24.05 \pm 0.05 \\
(15.00- \\
30.00)\end{array}$ & $\begin{array}{c}37.00 \pm 0.85 \\
(35.00- \\
45.00)\end{array}$ & $\begin{array}{c}4.05 \pm 1.05 \\
(3.00-8.00)\end{array}$ & $\begin{array}{c}7.75 \pm 0.05 \\
(3.00-12.00)\end{array}$ \\
\hline $\begin{array}{l}\text { JULY I } \\
2018\end{array}$ & $\begin{array}{l}20.75 \pm 1.45 \\
(15.0-26.00)\end{array}$ & $\begin{array}{c}35.80 \pm 3.04 \\
(25.00-38.00)\end{array}$ & $\begin{array}{c}35.45 \pm 3.50 \\
(20.00- \\
35.00)\end{array}$ & $\begin{array}{c}36.45 \pm 2.70 \\
(20.00- \\
39.00)\end{array}$ & $\begin{array}{c}20.65 \pm 1.50 \\
(15.00- \\
25.00)\end{array}$ & $\begin{array}{c}18.50 \pm 0.80 \\
(10.00- \\
25.00)\end{array}$ & $\begin{array}{c}25.50 \pm 0.04 \\
(20.00- \\
30.00)\end{array}$ & $\begin{array}{c}39.40 \pm 0.50 \\
(35.00- \\
45.00)\end{array}$ & $\begin{array}{c}5.50 \pm 1.04 \\
(3.00-9.00)\end{array}$ & $\begin{array}{c}7.80 \pm 0.05 \\
(2.00-9.00)\end{array}$ \\
\hline $\begin{array}{l}\text { JULY II } \\
2018\end{array}$ & $\begin{array}{l}30.45 \pm 0.90 \\
(26.00-38.00)\end{array}$ & $\begin{array}{c}30.40 \pm 1.02 \\
(20.00-40.00)\end{array}$ & $\begin{array}{c}32.00 \pm 6.50 \\
(20.00- \\
38.00)\end{array}$ & $\begin{array}{c}34.45 \pm 3.50 \\
(25.00- \\
50.00)\end{array}$ & $\begin{array}{c}16.90 \pm 2.50 \\
(5.00-18.00)\end{array}$ & $\begin{array}{c}14.50 \pm 0.70 \\
(2.00-15.00)\end{array}$ & $\begin{array}{c}28.00 \pm 0.01 \\
(20.00- \\
35.00)\end{array}$ & $\begin{array}{c}40.00 \pm 2.55 \\
(30.00- \\
45.00)\end{array}$ & $\begin{array}{c}6.00 \pm 1.01 \\
(3.00-10.00)\end{array}$ & $\begin{array}{c}8.00 \pm 0.05 \\
(3.00-11.00)\end{array}$ \\
\hline AUG. I 2018 & $\begin{array}{l}36.43 \pm 0.40 \\
(30.50-42.50)\end{array}$ & $\begin{array}{c}25.45 \pm 2.05 \\
(20.00-30.00)\end{array}$ & $\begin{array}{c}27.45 \pm 4.50 \\
(20.00- \\
38.00)\end{array}$ & $\begin{array}{c}27.35 \pm 4.50 \\
(15.00- \\
30.00)\end{array}$ & $\begin{array}{c}13.25 \pm 2.50 \\
(6.00-15.00)\end{array}$ & $\begin{array}{c}12.75 \pm 1.50 \\
(8.00-25.00)\end{array}$ & $\begin{array}{c}30.45 \pm 0.02 \\
(20.00- \\
35.00)\end{array}$ & $\begin{array}{c}43.00 \pm 1.20 \\
(35.00- \\
50.00)\end{array}$ & $\begin{array}{c}6.45 \pm 1.02 \\
(3.00-8.00)\end{array}$ & $\begin{array}{c}8.50 \pm 0.05 \\
(4.00-12.00)\end{array}$ \\
\hline $\begin{array}{l}\text { AUG. II } \\
2018\end{array}$ & $\begin{array}{l}38.25 \pm 1.30 \\
(35.00-47.80)\end{array}$ & $\begin{array}{c}22.30 \pm 2.03 \\
(20.00-30.00)\end{array}$ & $\begin{array}{c}25.90 \pm 3.50 \\
(20.00- \\
35.00)\end{array}$ & $\begin{array}{c}25.90 \pm 2.50 \\
(20.00- \\
30.00)\end{array}$ & $\begin{array}{c}12.25 \pm 1.50 \\
(8.00-20.00)\end{array}$ & $\begin{array}{c}11.35 \pm 2.50 \\
(8.00-25.00)\end{array}$ & $\begin{array}{c}3.35 \pm 0.05 \\
(1.00-6.00)\end{array}$ & $\begin{array}{c}8.00 \pm 0.75 \\
(3.00-10.00)\end{array}$ & $\begin{array}{c}0.35 \pm 0.05 \\
(0.00-1.50)\end{array}$ & $\begin{array}{c}2.50 \pm 0.05 \\
(2.00-3.00)\end{array}$ \\
\hline
\end{tabular}




\begin{tabular}{|c|c|c|c|c|c|c|c|c|c|c|}
\hline & $(32.00-53.00)$ & $(20.00-30.00)$ & $\begin{array}{l}(20.00- \\
36.00)\end{array}$ & $\begin{array}{l}(15.00- \\
35.00)\end{array}$ & $(8.00-20.00)$ & $(6.00-20.00)$ & $(1.00-3.00)$ & $(2.00-5.00)$ & $(0.00-1.50)$ & $(2.00-3.00)$ \\
\hline SEP. II 2018 & $\begin{array}{l}42.63 \pm 1.65 \\
(25.00-55.000\end{array}$ & $\begin{array}{c}20.10 \pm 4.05 \\
(20.00-25.00)\end{array}$ & $\begin{array}{c}20.75 \pm 2.50 \\
(15.00- \\
30.00)\end{array}$ & $\begin{array}{c}19.25 \pm 3.50 \\
(10.00- \\
25.00)\end{array}$ & $\begin{array}{c}10.45 \pm 2.50 \\
(9.00-25.00)\end{array}$ & $\begin{array}{c}8.25 \pm 3.50 \\
(5.00-15.00)\end{array}$ & $0.00 \pm 0.00$ & 00.00 & $0.00 \pm 0.00$ & 00.00 \\
\hline OCT.I 2018 & $\begin{array}{l}47.15 \pm 2.50 \\
(36.00-58.00)\end{array}$ & $\begin{array}{c}18.50 \pm 1.01 \\
(20.00-25.00)\end{array}$ & $\begin{array}{c}17.45 \pm 4.50 \\
(15.00- \\
20.00)\end{array}$ & $\begin{array}{c}18.75 \pm 2.50 \\
(10.00- \\
25.00)\end{array}$ & $\begin{array}{c}9.35 \pm 2.50 \\
(7.00-25.00)\end{array}$ & $\begin{array}{c}8.00 \pm 2.50 \\
(4.00-15.00)\end{array}$ & $0.00 \pm 0.00$ & 00.00 & $0.00 \pm 0.00$ & 00.00 \\
\hline OCT. II 2018 & $\begin{array}{l}53.16 \pm 2.30 \\
(48.50-60.70)\end{array}$ & $\begin{array}{c}17.75 \pm 3.05 \\
(20.00-20.00)\end{array}$ & $\begin{array}{c}17.00 \pm 3.50 \\
(10.00- \\
20.00)\end{array}$ & $\begin{array}{c}12.35 \pm 2.70 \\
(10.00- \\
15.00)\end{array}$ & $\begin{array}{c}9.25 \pm 2.50 \\
(5.25-17.00)\end{array}$ & $\begin{array}{c}7.45 \pm 1.00 \\
(2.00-12.00)\end{array}$ & $0.00 \pm 0.00$ & 00.00 & $0.00 \pm 0.00$ & 00.00 \\
\hline NOV. I 2018 & $\begin{array}{l}49.16 \pm 3.32 \\
(42.00-54.00)\end{array}$ & $\begin{array}{c}16.90 \pm 1.06 \\
(10.00-25.00)\end{array}$ & $\begin{array}{c}16.45 \pm 4.10 \\
(10.00- \\
18.00)\end{array}$ & $\begin{array}{c}8.35 \pm 3.50 \\
(6.00-12.00)\end{array}$ & $\begin{array}{c}8.56 \pm 2.50 \\
(5.00-15.00)\end{array}$ & $\begin{array}{c}6.75 \pm 2.50 \\
(2.00-12.00)\end{array}$ & $0.00 \pm 0.00$ & 00.00 & $0.00 \pm 0.00$ & 00.00 \\
\hline $\begin{array}{l}\text { NOV. II } \\
2018\end{array}$ & $\begin{array}{l}44.53 \pm 01.50 \\
(44.00-45.00)\end{array}$ & $\begin{array}{c}14.05 \pm 2.03 \\
(11.00-23.00)\end{array}$ & $\begin{array}{c}13.45 \pm 4.50 \\
(10.00- \\
20.00)\end{array}$ & $\begin{array}{c}6.90 \pm 2.50 \\
(3.00-10.00)\end{array}$ & $\begin{array}{c}7.25 \pm 2.50 \\
(6.00-15.00)\end{array}$ & $\begin{array}{c}5.75 \pm 1.50 \\
(2.00-9.00)\end{array}$ & $0.00 \pm 0.00$ & 00.00 & $0.00 \pm 0.00$ & 00.00 \\
\hline DEC. I 2018 & $\begin{array}{l}26.25 \pm 1.45 \\
(25.50-26.25)\end{array}$ & $\begin{array}{c}7.00 \pm 0.00 \\
(5.00-10.00)\end{array}$ & $\begin{array}{c}12.75 \pm 1.75 \\
(10.00- \\
20.00)\end{array}$ & $\begin{array}{c}5.65 \pm 4.50 \\
(5.00-8.00)\end{array}$ & $\begin{array}{c}5.25 \pm 2.50 \\
(5.00-9.00)\end{array}$ & $\begin{array}{c}5.00 \pm 0.50 \\
(2.00-10.00)\end{array}$ & $0.00 \pm 0.00$ & 00.00 & $0.00 \pm 0.00$ & 00.00 \\
\hline $\begin{array}{l}\text { DEC. II } \\
2018\end{array}$ & $\begin{array}{l}12.25 \pm 1.08 \\
(10.00-22.00)\end{array}$ & $00.00 \pm 0.00$ & $0.00 \pm 0.00$ & $0.00 \pm 0.00$ & 00.0 & $00.00 \pm 0.00$ & $0.00 \pm 0.00$ & 00.00 & $0.00 \pm 0.00$ & 00.00 \\
\hline JAN. I 2019 & $\begin{array}{l}10.60 \pm 1.26 \\
(8.50-15.00)\end{array}$ & $0.00 \pm 0.00$ & $0.00 \pm 0.00$ & $0.00 \pm 0.00$ & 00.00 & $00.00 \pm 0.00$ & $0.00 \pm 0.00$ & 00.00 & $0.00 \pm 0.00$ & 00.00 \\
\hline JAN.II 2019 & $\begin{array}{l}12.07 \pm 0.45 \\
(9.00-17.00)\end{array}$ & $0.00 \pm 0.00$ & $0.00 \pm 0.00$ & $0.00 \pm 0.00$ & 00.00 & $00.00 \pm 0.00$ & $\begin{array}{c}10.00 \pm 1.00 \\
(5.00-15.00)\end{array}$ & $\begin{array}{c}17.45 \pm 1.90 \\
(15.00- \\
20.00)\end{array}$ & $\begin{array}{c}2.25 \pm 0.05 \\
(1.50-4.00)\end{array}$ & $\begin{array}{c}5.00 \pm 1.00 \\
(3.00-6.00)\end{array}$ \\
\hline FEB. I 2019 & $\begin{array}{l}12.90 \pm 0.50 \\
(7.00-15.50)\end{array}$ & $0.00 \pm 0.00$ & $0.00 \pm 0.00$ & $0.00 \pm 0.00$ & 00.00 & $00.00 \pm 0.00$ & $\begin{array}{c}13.50 \pm 1.50 \\
(10.00- \\
18.00)\end{array}$ & $\begin{array}{c}20.38 \pm 2.67 \\
(17.00- \\
\left.22.00 \_\right)\end{array}$ & $\begin{array}{c}2.75 \pm 0.06 \\
(1.75-4.00)\end{array}$ & $\begin{array}{c}5.20 \pm 0.05 \\
(3.00-7.00)\end{array}$ \\
\hline FEB. II 2019 & $\begin{array}{l}10.00 \pm 0.50 \\
(9.00-10.50)\end{array}$ & $0.00 \pm 0.00$ & $0.00 \pm 0.00$ & $0.00 \pm 0.00$ & 00.00 & $00.00 \pm 0.00$ & $\begin{array}{c}15.00 \pm 2.00 \\
(12.00- \\
18.00)\end{array}$ & $\begin{array}{c}22.65 \pm 1.00 \\
15.00- \\
25.00)\end{array}$ & $\begin{array}{c}3.90 \pm 0.05 \\
(2.00-5.00)\end{array}$ & $\begin{array}{c}5.25 \pm 1.00 \\
(3.00-4.00)\end{array}$ \\
\hline $\begin{array}{l}\text { MARCH I } \\
2019\end{array}$ & $\begin{array}{l}10.14 \pm 0.30 \\
(9.00-13.00)\end{array}$ & $\begin{array}{c}0.00 \pm 0.00 \\
(00.00-00.00)\end{array}$ & $0.00 \pm 0.00$ & $0.00 \pm 0.00$ & 00.00 & $00.00 \pm 0.00$ & $\begin{array}{c}18.00 \pm 2.50 \\
(12.00- \\
25.00)\end{array}$ & $\begin{array}{c}25.90 \pm 3.75 \\
20.00- \\
28.00)\end{array}$ & $\begin{array}{c}4.20 \pm 0.02 \\
(2.00-5.00)\end{array}$ & $\begin{array}{c}5.75 \pm 0.05 \\
(2.00-9.00)\end{array}$ \\
\hline MARCH II & $10.34 \pm 1.40$ & $12.70 \pm 2.08$ & $11.45 \pm 2.50$ & $12.15 \pm 2.50$ & $12.25 \pm 2.50$ & $18.25 \pm 2.50$ & $20.00 \pm 2.00$ & $28.45 \pm 2.80$ & $4.25 \pm 0.03$ & $5.80 \pm 1.00$ \\
\hline
\end{tabular}




\begin{tabular}{|c|c|c|c|c|c|c|c|c|c|c|}
\hline 2019 & $(10.0-11.00)$ & $(10.00-25.00)$ & $\begin{array}{l}(9.00- \\
18.00)\end{array}$ & $(5.00-15.00)$ & $(9.00-15.00)$ & $\begin{array}{l}(15.00- \\
25.00)\end{array}$ & $\begin{array}{l}(15.00- \\
25.00)\end{array}$ & $\begin{array}{l}24.00- \\
30.00)\end{array}$ & $(2.00-5.00)$ & $(2.00-10.00)$ \\
\hline $\begin{array}{l}\text { APRIL I } \\
2019\end{array}$ & $\begin{array}{l}11.26 \pm 0.50 \\
(11.00-12.00)\end{array}$ & $\begin{array}{c}14.10 \pm 1.04 \\
(10.00-20.00)\end{array}$ & $\begin{array}{c}12.75 \pm 2.50 \\
(10.00- \\
15.00)\end{array}$ & $\begin{array}{c}14.75 \pm 4.50 \\
(5.00-25.00)\end{array}$ & $\begin{array}{c}14.25 \pm 2.50 \\
(9.00-20.00)\end{array}$ & $\begin{array}{c}20.35 \pm 1.50 \\
(17.00- \\
25.00)\end{array}$ & $\begin{array}{c}23.00 \pm 1.00 \\
(18.00- \\
28.00)\end{array}$ & $\begin{array}{c}31.75 \pm 2.00 \\
(25.00- \\
32.00)\end{array}$ & $\begin{array}{c}4.30 \pm 0.04 \\
(2.00-5.00)\end{array}$ & $\begin{array}{c}6.00 \pm 0.05 \\
(3.00-12.00)\end{array}$ \\
\hline $\begin{array}{l}\text { APRIL II } \\
2019\end{array}$ & $\begin{array}{l}11.90 \pm 1.50 \\
(10.00-13.0)\end{array}$ & $\begin{array}{c}15.60 \pm 3.02 \\
(15.00-20.00)\end{array}$ & $\begin{array}{c}17.65 \pm 2.50 \\
(15.00- \\
20.00)\end{array}$ & $\begin{array}{c}16.85 \pm 3.50 \\
(15.00- \\
25.00)\end{array}$ & $\begin{array}{c}16.25 \pm 2.50 \\
(9.00-20.00)\end{array}$ & $\begin{array}{c}20.75 \pm 2.50 \\
(18.00- \\
25.00)\end{array}$ & $\begin{array}{c}25.80 \pm 1.00 \\
(20.00- \\
28.00)\end{array}$ & $\begin{array}{c}33.25 \pm 2.55 \\
(25.00- \\
33.00)\end{array}$ & $\begin{array}{c}4.30 \pm 0.01 \\
(2.00-5.00)\end{array}$ & $\begin{array}{c}6.00 \pm 0.05 \\
(3.00-12.00)\end{array}$ \\
\hline
\end{tabular}

I - First fortnight, II- Second fortnight*Figure in the parenthesis are range values*Each figure is the mean of 50 sampling units \pm S.D. 
Table.4 Infestation of leaf miner in Pongamiapinnata(L.) Pierre at Bhubaneswar (2018-2019)

\begin{tabular}{|c|c|c|c|c|c|}
\hline $\begin{array}{l}\text { Period of } \\
\text { observation }\end{array}$ & $\begin{array}{l}\text { leaf miner } \\
\text { mean \% plant } \\
\text { infestation }\end{array}$ & $\begin{array}{l}\text { Mean \% } \\
\text { compound leaf } \\
\text { infestation }\end{array}$ & $\begin{array}{l}\text { Mean blotch } \\
\text { intensity per } \\
\text { compound leaves }\end{array}$ & $\begin{array}{l}\text { Mean \% of } \\
\text { blotches } \\
\text { harbouring miner } \\
\text { larvae }\end{array}$ & $\begin{array}{l}\text { Mean larval } \\
\text { population } \\
\text { density per leaf }\end{array}$ \\
\hline MAY I, 2018 & $\begin{array}{l}10.70 \pm 1.45 \\
(8.00-12.00)\end{array}$ & $\begin{array}{l}4.75 \pm 1.03 \\
(3.00-7.00)\end{array}$ & $\begin{array}{l}3.70 \pm 1.04 \\
(2.00-6.00)\end{array}$ & $\begin{array}{l}22.54 \pm 5.50 \\
(15.00-25.00)\end{array}$ & $\begin{array}{l}1.50 \pm 0.50 \\
(1.00-2.00)\end{array}$ \\
\hline MAY II 2018 & $\begin{array}{l}13.85 \pm 1.45 \\
10.00-15.00)\end{array}$ & $\begin{array}{l}6.25 \pm 1.03 \\
(3.00-9.00)\end{array}$ & $\begin{array}{l}4.25 \pm 1.03 \\
(3.00-7.00)\end{array}$ & $\begin{array}{l}27.54 \pm 5.50 \\
(20.00-30.00)\end{array}$ & $\begin{array}{l}1.50 \pm 0.50 \\
(1.00-2.00)\end{array}$ \\
\hline JUNE I 2018 & $\begin{array}{l}15.75 \pm 1.45 \\
(10.00-18.00)\end{array}$ & $\begin{array}{l}7.50 \pm 1.03 \\
(4.00-10.00)\end{array}$ & $\begin{array}{l}5.00 \pm 3.02 \\
(3.00-10.00)\end{array}$ & $\begin{array}{l}37.54 \pm 5.50 \\
(35.00-45.00)\end{array}$ & $\begin{array}{l}1.50 \pm 0.50 \\
(1.00-2.00)\end{array}$ \\
\hline JUNE II 2018 & $\begin{array}{l}20.45 \pm 1.45 \\
(15.00-25.00)\end{array}$ & $\begin{array}{l}10.00 \pm 1.03 \\
(8.50-12.00)\end{array}$ & $\begin{array}{l}5.70 \pm 2.01 \\
(3.00-10.00)\end{array}$ & $\begin{array}{l}57.54 \pm 5.50 \\
(50.00-65.00)\end{array}$ & $\begin{array}{l}1.50 \pm 0.50 \\
(1.00-2.00)\end{array}$ \\
\hline JULY I 2018 & $\begin{array}{l}65.00 \pm 5.50 \\
(60.00-70.00)\end{array}$ & $\begin{array}{l}20.00 \pm 1.03 \\
(15.00-23.00)\end{array}$ & $\begin{array}{l}6.60 \pm 1.05 \\
(3.00-8.00)\end{array}$ & $\begin{array}{l}90.00 \pm 5.50 \\
(80.00-100.00)\end{array}$ & $\begin{array}{l}1.50 \pm 0.50 \\
(1.00-2.00)\end{array}$ \\
\hline JULY II 2018 & $\begin{array}{l}70.00 \pm 5.50 \\
(65.00-75.00)\end{array}$ & $\begin{array}{l}25.66 \pm 3.45 \\
(15.00-30.00)\end{array}$ & $\begin{array}{l}8.30 \pm 1.07 \\
(4.00-9.00)\end{array}$ & $\begin{array}{l}85.00 \pm 5.50 \\
(70.00-90.00)\end{array}$ & $\begin{array}{l}1.50 \pm 0.50 \\
(1.00-2.00)\end{array}$ \\
\hline AUG. I 2018 & $\begin{array}{l}80.00 \pm 5.50 \\
(70.00-90.00)\end{array}$ & $\begin{array}{l}30.66 \pm 3.45 \\
(25.00-35.00)\end{array}$ & $\begin{array}{l}13.25 \pm 2.04 \\
(10.00-16.00)\end{array}$ & $\begin{array}{l}80.00 \pm 5.50 \\
(70.00-85.00)\end{array}$ & $\begin{array}{l}1.50 \pm 0.50 \\
(1.00-3.00)\end{array}$ \\
\hline AUG. II 2018 & $\begin{array}{l}82.00 \pm 5.50 \\
(70.00-85.00)\end{array}$ & $\begin{array}{l}50.66 \pm 3.45 \\
(45.00-55.00)\end{array}$ & $\begin{array}{l}15.80 \pm 1.01 \\
(12.00-18.00)\end{array}$ & $\begin{array}{l}70.00 \pm 5.50 \\
(65.00-75.00)\end{array}$ & $\begin{array}{l}1.50 \pm 0.50 \\
(1.00-2.00)\end{array}$ \\
\hline SEP. I 2018 & $\begin{array}{l}84.00 \pm 5.50 \\
(80.00-90.00)\end{array}$ & $\begin{array}{l}56.66 \pm 3.45 \\
(50.00-60.00)\end{array}$ & $\begin{array}{l}18.65 \pm 0.03 \\
(15.00-20 . .00)\end{array}$ & $\begin{array}{l}50.00 \pm 5.50 \\
40.00-55.00\end{array}$ & $\begin{array}{l}1.50 \pm 0.50 \\
(1.00-3.00)\end{array}$ \\
\hline SEP. II 2018 & $\begin{array}{l}85.00 \pm 5.50 \\
(80.00-90.00)\end{array}$ & $\begin{array}{l}60.66 \pm 3.45 \\
(60.00-70.00)\end{array}$ & $\begin{array}{l}19.00 \pm 0.05 \\
(16.00-22.00)\end{array}$ & $\begin{array}{l}52.00 \pm 5.50 \\
(45.00-55.00)\end{array}$ & $\begin{array}{l}1.50 \pm 0.50 \\
(1.00-3.00)\end{array}$ \\
\hline OCT.I 2018 & $\begin{array}{l}89.00 \pm 5.50 \\
(80.00-95.00)\end{array}$ & $\begin{array}{l}66.66 \pm 3.45 \\
(60.00-70.00)\end{array}$ & $\begin{array}{l}22.60 \pm 1.07 \\
(18.00-24.00)\end{array}$ & $\begin{array}{l}28.00 \pm 5.50 \\
(25.00-30.00)\end{array}$ & $\begin{array}{l}1.50 \pm 0.50 \\
(1.00-3.00)\end{array}$ \\
\hline OCT. II 2018 & $\begin{array}{l}70.00 \pm 5.50 \\
(60.00-80.00)\end{array}$ & $\begin{array}{l}60.00 \pm 2.45 \\
(55.00-65.00)\end{array}$ & $\begin{array}{l}22.05 \pm 2.03 \\
(18.00-25.00)\end{array}$ & $\begin{array}{l}21.00 \pm 5.50 \\
(15.00-25.00)\end{array}$ & $\begin{array}{l}1.50 \pm 0.50 \\
(1.00-2.00)\end{array}$ \\
\hline NOV. I 2018 & $\begin{array}{l}40.00 \pm 5.50 \\
(30.00-45.00)\end{array}$ & $\begin{array}{l}40.00 \pm 3.45 \\
(35.00-45.00)\end{array}$ & $\begin{array}{l}20.35 \pm 3.05 \\
(19.00-26.00)\end{array}$ & $\begin{array}{l}18.33 \pm 5.50 \\
(15.00-25.00)\end{array}$ & $\begin{array}{l}1.50 \pm 0.50 \\
(1.00-3.00)\end{array}$ \\
\hline NOV. II 2018 & $\begin{array}{l}25.00 \pm 5.50 \\
(20.00-30.00)\end{array}$ & $\begin{array}{l}25.00 \pm 1.45 \\
(20.00-35.00)\end{array}$ & $\begin{array}{l}13.35 \pm 0107 \\
(10.00-15.00)\end{array}$ & $\begin{array}{l}13.33 \pm 5.50 \\
(10.00-20.00)\end{array}$ & $\begin{array}{l}1.50 \pm 0.50 \\
(1.00-2.00)\end{array}$ \\
\hline DEC. I 2018 & $\begin{array}{l}15.00 \pm 5.50 \\
(10.00-20.00)\end{array}$ & $\begin{array}{l}12.25 \pm 3.45 \\
(10.00-15.00)\end{array}$ & $\begin{array}{l}1.50 \pm 2.04 \\
(1.00-3.00)\end{array}$ & $\begin{array}{l}5.00 \pm 5.50 \\
(4.00-11.00)\end{array}$ & $\begin{array}{l}1.50 \pm 0.50 \\
(1.00-2.00)\end{array}$ \\
\hline DEC. II 2018 & $0.00 \pm 0.00$ & $0.00 \pm 0.00$ & $0.00 \pm 0.00$ & $0.00 \pm 0.00$ & $0.00 \pm 0.00$ \\
\hline JAN. I 2019 & $0.00 \pm 0.00$ & $0.00 \pm 0.00$ & $0.00 \pm 0.00$ & $0.00 \pm 0.00$ & $0.00 \pm 0.00$ \\
\hline JAN.II 2019 & $0.00 \pm 0.00$ & $0.00 \pm 0.00$ & $0.00 \pm 0.00$ & $0.00 \pm 0.00$ & $0.00 \pm 0.00$ \\
\hline FEB. I 2019 & $0.00 \pm 0.00$ & $0.00 \pm 0.00$ & $0.00 \pm 0.00$ & $0.00 \pm 0.00$ & $0.00 \pm 0.00$ \\
\hline FEB. II 2019 & $0.00 \pm 0.00$ & $0.00 \pm 0.00$ & $0.00 \pm 0.00$ & $0.00 \pm 0.00$ & $0.00 \pm 0.00$ \\
\hline MARCH I 2019 & $0.00 \pm 0.00$ & $0.00 \pm 0.00$ & $0.00 \pm 0.00$ & $0.00 \pm 0.00$ & $0.00 \pm 0.00$ \\
\hline $\begin{array}{l}\text { MARCH II } \\
2019\end{array}$ & $\begin{array}{l}5.00 \pm 1.45 \\
(2.008 .00)\end{array}$ & $\begin{array}{l}3.25 \pm 3.45 \\
(2.00-5.00)\end{array}$ & $\begin{array}{l}3.40 \pm 2.05 \\
(2.00-6.00)\end{array}$ & $\begin{array}{l}12.75 \pm 5.50 \\
(9.00-15.00)\end{array}$ & $\begin{array}{l}1.50 \pm 0.50 \\
(1.00-2.00)\end{array}$ \\
\hline APRIL I 2019 & $\begin{array}{l}8.25 \pm 1.45 \\
(5.00-12.00)\end{array}$ & $\begin{array}{l}5.25 \pm 1.45 \\
(3.00-8.00)\end{array}$ & $\begin{array}{l}3.40 \pm 1.03 \\
(2.00-6.00)\end{array}$ & $\begin{array}{l}15.65 \pm 5.50 \\
(9.00-18.00)\end{array}$ & $\begin{array}{l}1.50 \pm 0.50 \\
(1.00-2.00)\end{array}$ \\
\hline APRIL II 2019 & $\begin{array}{l}10.75 \pm 1.45 \\
(8.00-13.00)\end{array}$ & $\begin{array}{l}7.50 \pm 2.45 \\
(4.00-12.00)\end{array}$ & $\begin{array}{l}3.50 \pm 2.01 \\
(2.00-6.00)\end{array}$ & $\begin{array}{l}17.54 \pm 5.50 \\
(15.00-20.00)\end{array}$ & $\begin{array}{l}1.50 \pm 0.50 \\
(1.00-2.00)\end{array}$ \\
\hline
\end{tabular}

*Figure in the parenthesis are range values *Each figure is the mean of 50 sampling units \pm S.D 

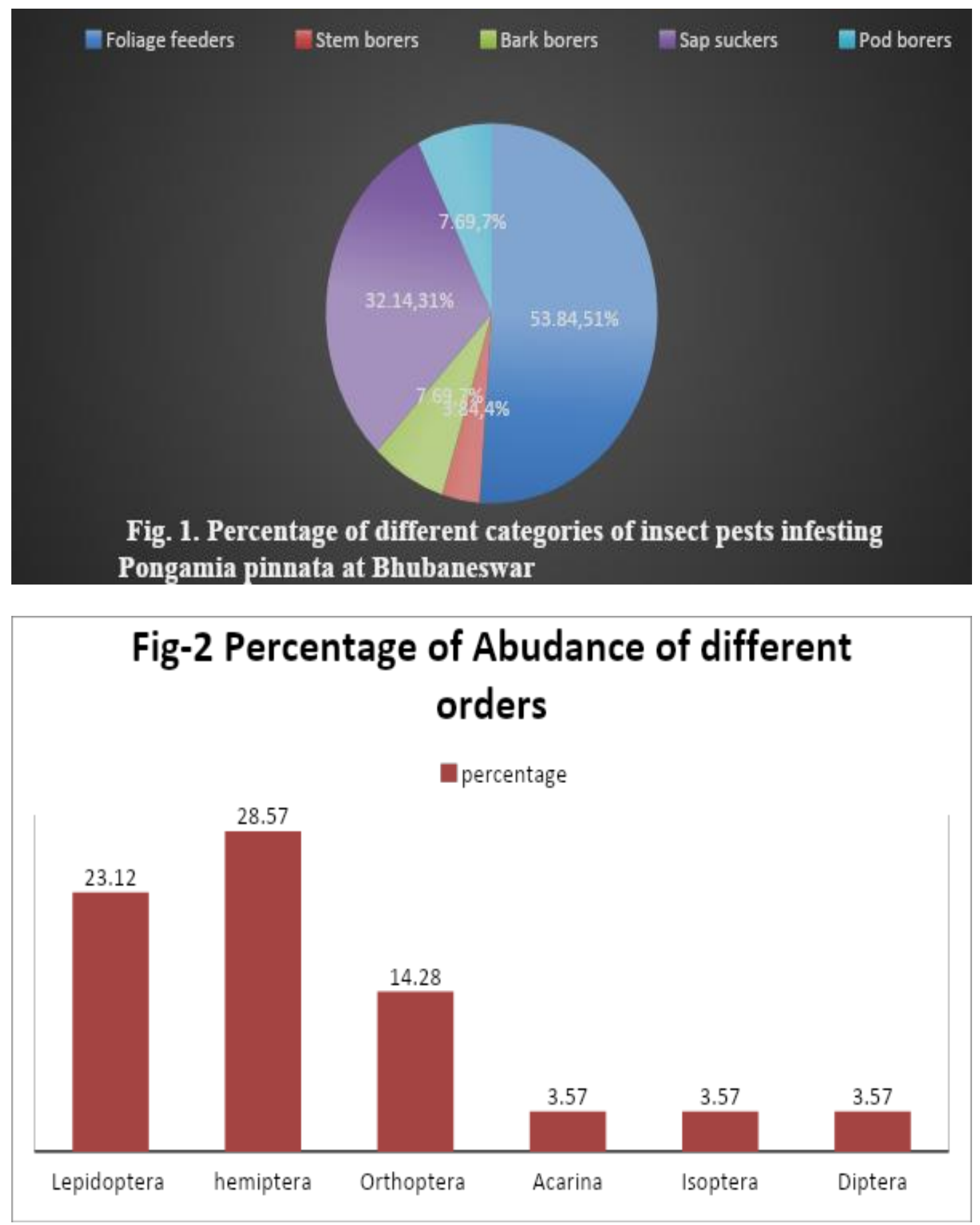
Fig.3 Insect and non insect pest of Pongamia pinnata

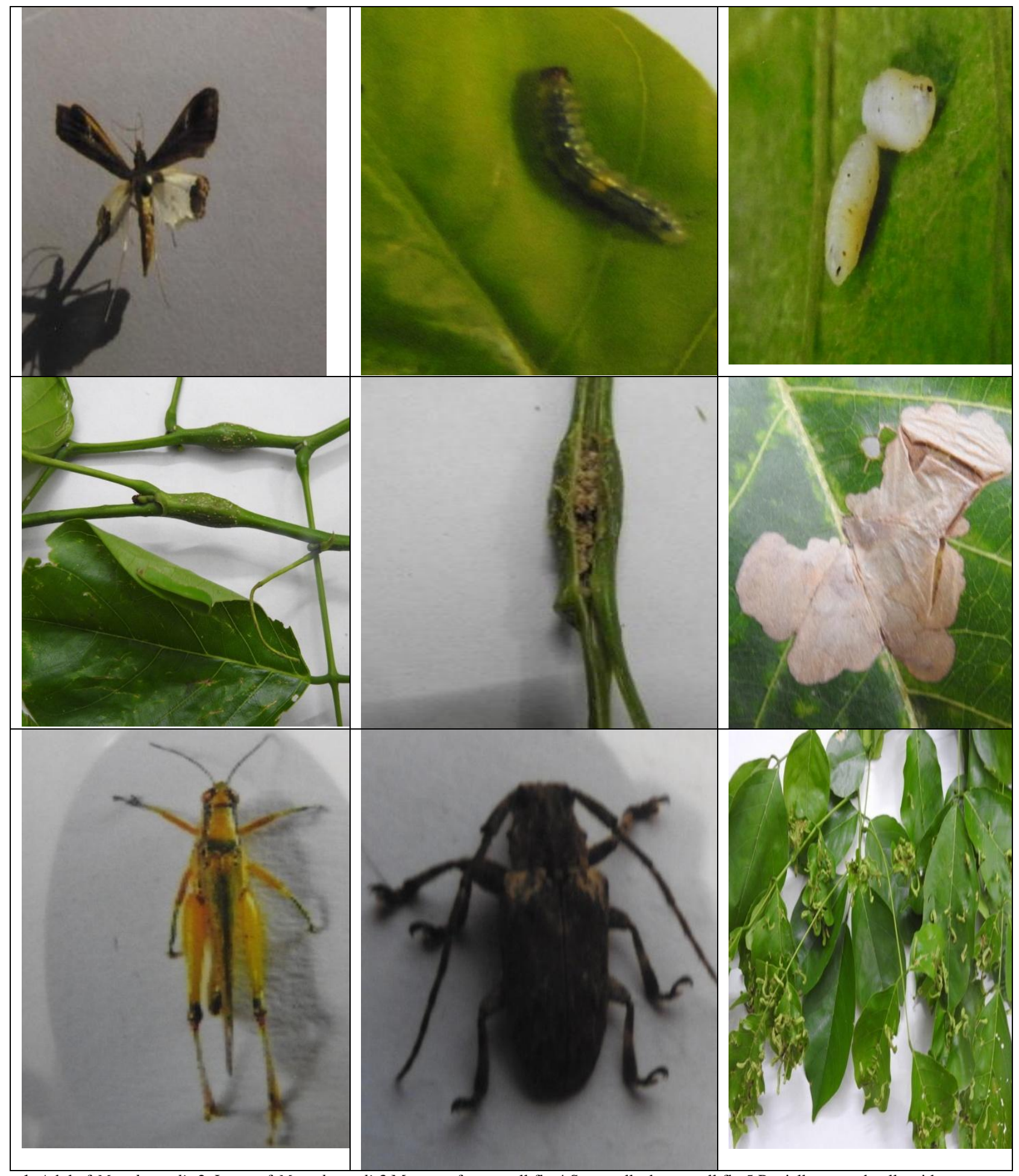

1. Adultof M.ambuanalis 2. Larva of M. ambuanalis3.Maggot of stem gall fly 4.Stem galls due to gall fly 5.Partially opened galls with maggots 6.Leaf blotch after minor emergence7. Grass hopper 8.Stem borer 8.Leaf galls due to eriophyid mite 


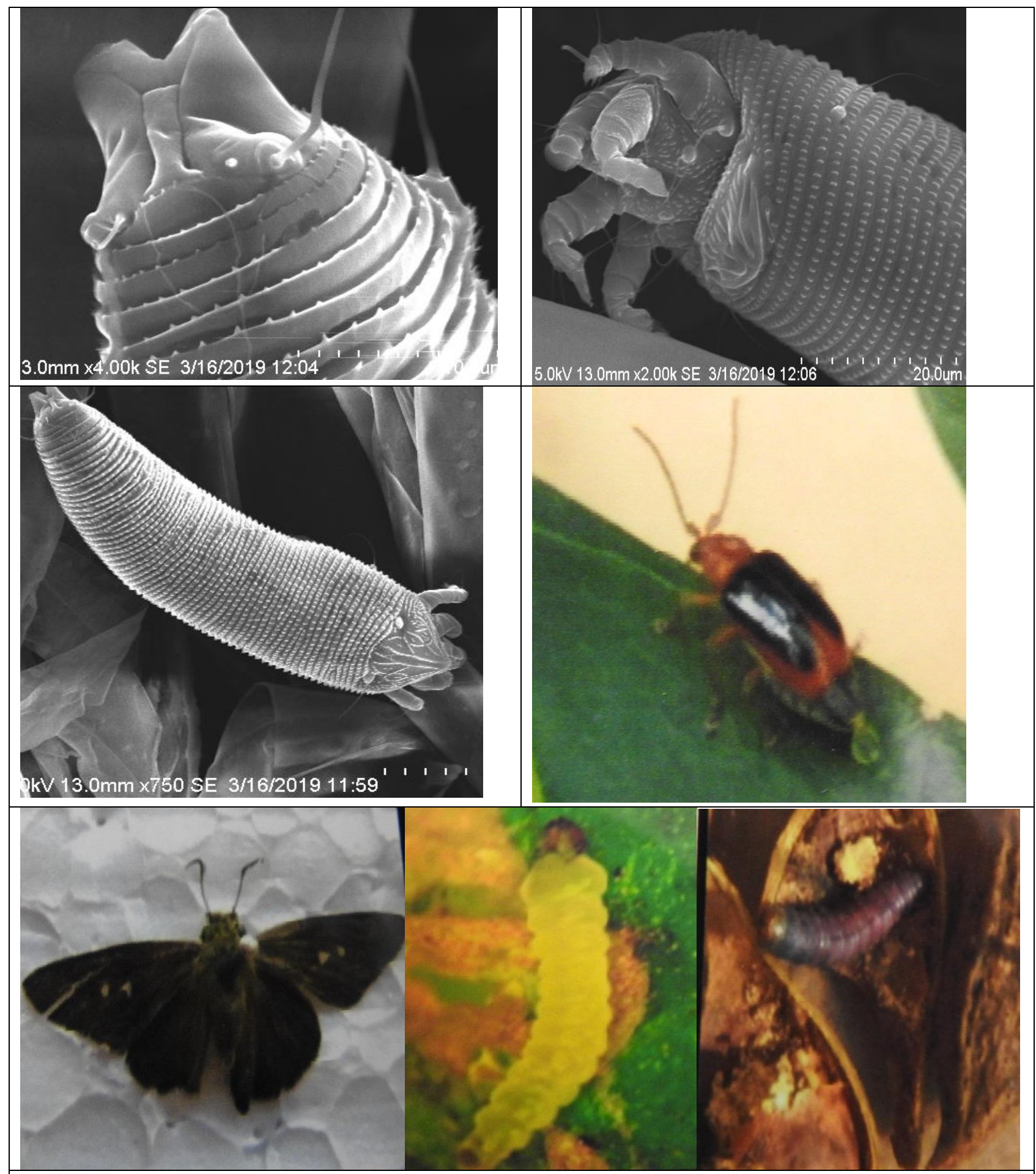

10.Posterior parts of mite alog with enlarged EMS image of bodyrings11.Mouth parts12. Structre oof mite enlarged EMS views13.Diabrotica sp 14 Adult of Hasora chromus15.Larvae of leaf minor16.Larva of Ephestia cautela17.Curetis thetis 18 Termite Coptotermus sp!9 Adult of gall making fly

Natural enemies-1.Eulophid parasitoid of leaf minor 2.Spotted ord weaver spider 3 two striped jumper spider 
Fig.4 Natural enemies of insect pests of Pongamia pinnata

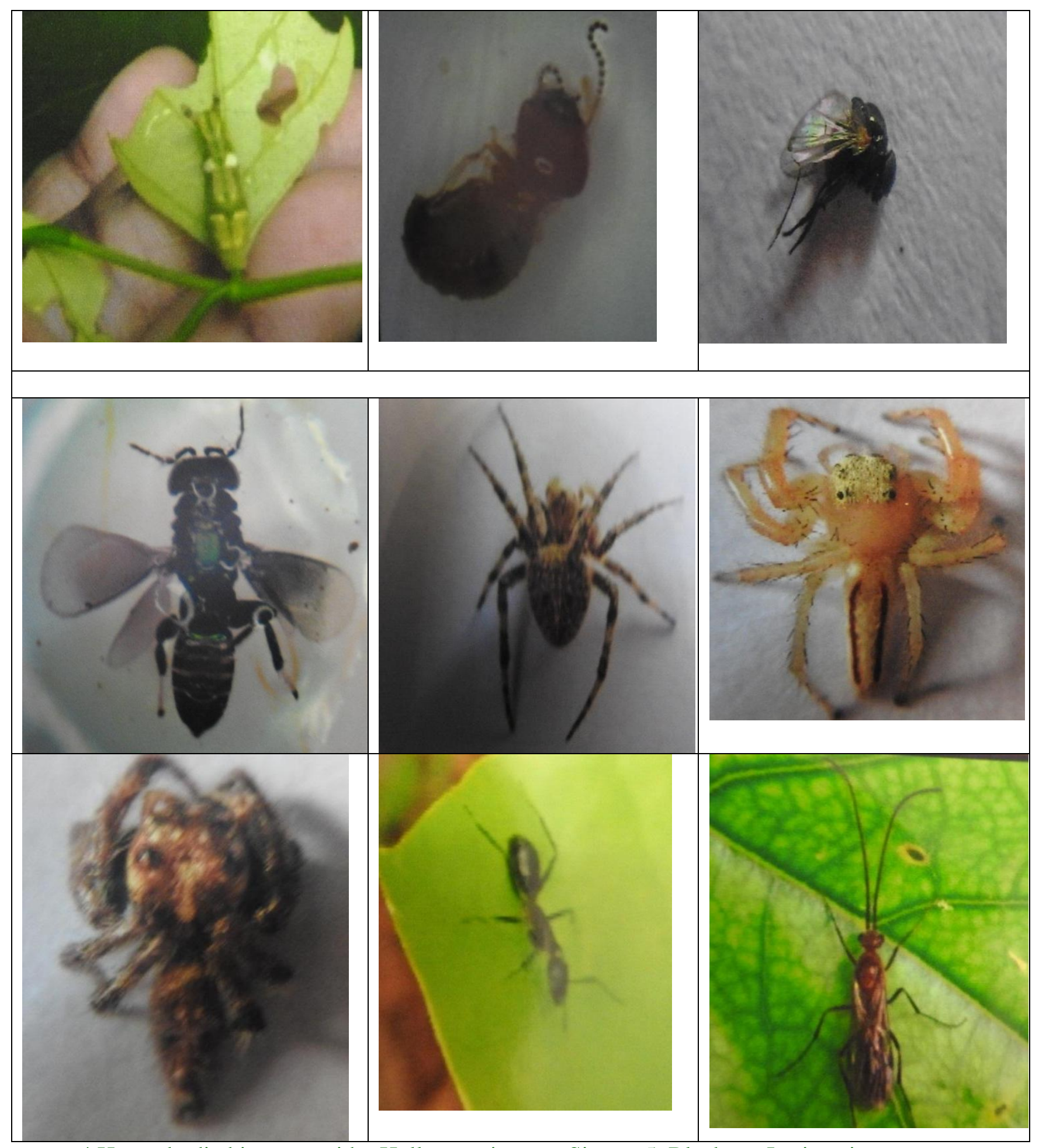

4.Heavy bodied jumper spider,Hyllus semicuprus Simon, 5. Black ant Lesius niger

6. Parasitoid unidentified 


\section{Infestation of leaf miner Lithocolitis virgulata Meyrick}

The mean percentage plant infestation due to leaf miner attack was varied from minimum of $15 \%$ infestation during $\left(1^{\text {st }}\right.$ fortnight of December 2018) to a maximum of $89.00 \%$ as recorded during first fortnight of October, 2018.(Table-4) Then no incidence was observed in fresh leaves up to first fortnight of March, 2019. Mean percentage of blotches harboring miner larvae varied from $5.00 \%\left(1^{\text {st }}\right.$ fortnight of December) to $80.00 \%$ during $2^{\text {nd }}$ fortnight of September and $1^{\text {st }}$ fortnight of October. A total of 28 species of insects including 1 species of Acarina are recorded to infest this plant which are categorized under 5 groups viz. i) Foliage feeders or defoliator ii) Stem borer iii) Bark borer iv)Sap feeder v) Seed feeder. The essentialities of present classification are that of Speight (2004).Out of the total species recorded $32.14 \%$ belongs to the order Lepidoptera, followed by $28.57 \%$ from Hemiptera, $14.28 \%$ each from Orthoptera and Coleoptera and 3.57\% each from Acarian, Isoptera and Diptera. A total of 12 species of natural enemies including 5 species of spiders were also recorded.. Leaf miner (Lithocolitis virgulata Meyrick) has been reported as the major pest of this plant in Delhi, Hyderabad and Jabalpur by Sushil Kumar (1992). However, Orwa et al., (2009) from Kenya reported the blotch miner in this plant as the major pest. The status of both the names is not clearly mentioned everywhere. Another related species of miner Acrocercops syngramma was reportedly attacking other plant of Cashew in several places of South India (Nair and Abrahim, 1982; Pillai,1979) but Fletcher (1919) had reported 70 species under Gracillaridae were be the leaf miners out of which 40 species were belonging to genus Acrocercops only. Workers like Lal et al., (1996) from Eastern U.P. and Singh (1990) also opined bark eating caterpillar, Inderbela quadrinotata to be the number one pest of this plant at their places. Sundararaj et al., (2005) reported a total of about 30 species of insects from Bangalore whereas Sahu et al., (2016) from Raipur and Seth (2017) from Jabalpur observed 24 and 10 species of insect pest infesting this plant respectively as. major pest. The status of both the names is not clearly mentioned everywhere. Reports of occurrence of leaf miner in other countries like Myanmar, Srilanka, Nepal, Pakistan is also available. Anonymous (2010). From Kenya and AfricaOrwa et al., (2009) reported 30 species of insect pests causing damage to Pongamia pinnata (L.)Pierre. Hasorachromus Cramer was also reported as the major pest of this plant during monsoon season by Harinath et al., (2012). Occurrence of leaf gall mite in this plant in causing gall incidence is also reported from other places like Kerala, Nasareem and Ramani (2014) and Bangalore, Rani et al., (2018). Sahu et al., (2015) from Raipur, Chavan and Saxena from South Gujarat (2012) and Sundararaj et al., (1984) from Bangalore, Haseeb et al., (2000) from Aligarh were also reported natural enemy complex of pests infesting this plant. However, detailed studies on extent of parasitization of any parasites on any specific pest have not been available from any sources. Perhaps because of large size and difficulty to work in height particularly effective sampling methods attract fewer workers to work in detail. But huge scale plantation in all the possible areas with increase in number of insects and non-insect pests will force to formulate the area specific IPM strategies in which use of bio control agent is the important one. Although classification of insects as per their mode of feeding is available for important timber yielding trees like Sal, Sissoo, teak, acacia etc., it is the first attempt in this country to categorize the documented insects infesting this plant, based upon their mode of feeding. Among the species recorded defoliators were comprising $54 \%$ of the total and this is 
followed by $31.14 \% \%$ of sap feeders, other species of insect categories comprised of $14.86 \%$ of the total recorded species.. Stem gall fly incidence was recorded throughout the investigation period except from September $2^{\text {nd }}$ fortnight to January $1^{\text {st }}$ fortnight and the infested branches were also either regenerating branches or side tender shoots of pole or medium age trees. More than one maggot were recovered from each gall and highest (8.50 maggots/gall) number of maggots were recovered during August $1^{\text {st }}$ fortnight, 2018.

The multilocular galls generally support one maggot in each chamber and adult flies come out of the gall by making holes. Pupation generally occurs inside the galls. The galls are formed by hypertrophy and hyperplasia of medullary and pith cells. Gall making flies and tehritids also possess the same characteristics (Johnston and Deneke, 2008). Being a general Dipteran more activity of this pest was found during rainy and post rainy season period. Population density fluctuation over a period of time is population dynamics which may be influenced by both the climatic and biotic factors. The men population density of Hasora chromus Cramer larva was found highest (19.80/plant) during October $1^{\text {st }}$ fortnight, 2018 and lowest was found during December $\quad 1^{\text {st }} \quad$ fortnight, $2018 \quad$ (1.95 larva/plant). However, during rest period of the year neither the larvae nor the eggs were reported. The availability is higher in post rainy period which was continued up to December $1^{\text {st }}$ fortnight. This is in full agreement with Harinath et al., (2012) from A.P. As observed from the present study the population is mostly confined to plants grown in swampy areas. Other skipper infesting paddy in Odisha made its significant presence in their major host plant paddy during the same period as reported by several workers Tripathy et al., (2016). Hasora chromus is a polyphagous pest and other reported host plants are Swietenia, Ricinus, Murraya, Pithecellobium, Castor etc. Suryanarayan et al., (2015) so during the period of unavailability it might be switched to any of its host plants or might undergo diapause or hibernation to spent winter which needs to studied in detail. Maruca ambionalis Felder is recently reported as a pest infesting this plant, but the appearance is for a short period The population of other related species Maruca vitrata is recorded from its major host plant pigeon pea during the pod development and flowering period i.e. October to December in major cultivation areas.

\section{Acknowledgement}

The authors are highly thankful to the central instrumentation facility, OUAT Bhubaneswar for providing facilities for use of scanning electron microscope. The help rendered by HOD, Entomology for identifying the specimens is also duly acknowledged.

\section{References}

Anonymos .2010.National Research Centre for Agroforestry, Jhansi. Uttar Pradesh.

Chavan SM and Saxena S.2012.Natural parasitization of Karanj defoliator , Glyphodes negatalis, Walker (Lepidoptera:Pyralidae): a newly reported insect pest of Karanja in South Gujurat, Medicinal Plant Research,2(4):18-20.

Devika Rani, Naik DJ and Jagdish KS 2018. Seasonal Incidence of Major Pests of pongamia (Milletia pinnata L.) in Nursery conditions. Int.J.Curr.Microbiol.App.Sci, 7(8):2337-2350.

Fletcher TU.1920.Life histories of Indian micro Lepidoptera, Memoirs of the Department Agriculture in India,6(6):151.

Haseeb M, Abbas SR, Srivastava RP and 
Sharma S.2000. Studies on insect pest of Kaanja, Annuals of Plant Protection, 8(1):85-88.

Harinath P, Kumar VP and Ramaana SP.2011. The Eco biology of common banded awl Hasora chromu sCramer (Lepidoptera:Rhopalocera; Satyridae) from Southern Andhra Pradesh, Bulletin of pure and Applied Sciences,31(1):3742.

Johnson PJ, Deneke Dl.2018.Biocontrol insects of Canada thistle in the northern great plains, $S D S U$ extension Special Series, paper 9.

Kumar Sushil. 1992.Epidemics of leaf miner (Lithocolletis virgulata) on Pongamia pinnata,Annals of Plant Protection Sciences,3(2): 164-190

Lal M N,Joshi GC and SS.(1996).Studies on the incidence of Inderbela spp. On B.stylophora and Karanj in Eastern UP, Progressive Horticulture, 28(1/2):66-71.

Nair GM and Abrahim C C. 1982. Insecticidal control of the cashew leaf miner Acrocercops syngrammaM, Pesticides, 16(9):19-20.

Nasareen, P N M, Ramani N.2014.Seasonal variation in the population density of the gall mit, Aceria pongamiae, keifer (Acari:Eriophyidae) within the leaf galls of Pongamia pinnata (L.), Journal of Entomology and Zoology Science, 2(3):126-130.

OrwaC,MutuaA, Kindt R, Jamnadass $\mathrm{R}$ and Simons A.2009.Agroforestry database. A tree reference and selection guide version 4.0.World Agroforestry Centre, Kenya $.491 \mathrm{p}$.

Patil SS, Sutar MV and SatheTV 2016. Diversity, Biology and Control of Insect Pests of teak Tectona grandis (Linnaeus) from Western, Maharashtra, Bio Life ,4(1):141-146

Pillai GB.1979. Pest control in cashew .Indian farming.28(12):2-28.

Sahu C, Ganguli JL, Ghirtlahre SK, Netam
CS, Painkra KL,2015.Screening of various provenances of karanja, Pongamia pinnata against hairy caterpillar, Euproctis sp. (Lepidoptera: Lymantriidae) at Raipur (Chattisgrh), International journal of Tourism Anthropology,33(2)529-532

Sahu C, Ganguli J L, Kerketta A, Painkra KL. 2016. Screening of Karanja, Pongomia pinnata L. against various insect pest under agro-silvi pattern of cropping system at Raipur(C.G), International journal of plant protection, 9(2):570577

Seth Vikas.2017.Screening of provenances of Pongamia pinnata(L.)Pierre against insect pest damage ,M..Sc(Ag),thesis submitted to Jawaharalal $\mathrm{N}$ ehruKrishi Viswa Vidyalaya, Jabalpur.84

Singh P.1990.Insect pest in Plantation of native tree sp. In India.Proceeding of IUFRO workshop pest and disease of forest plantation in the Asia Pasific Region FAO, Regional office for Asia Pacific, 45-55

Speight MR and Evans HF.2004.Integrated Pest Management Principles,Forestry Science Encyclopedia, 1,305-319

SundararajR, Rajamuthukrishna, Ramadevi OK.2005.Annotated list of insect pests of Pongomia pinnata (L.) Pierre in Indian. Annals of Forestry, 13:337-341.

SuryanarayanK,HarinathP,Venkata RM and Venkata RSP.2015.Life cycle of commonbanded awl Hasora chromus Cramer(Lepidoptera:Rhopalocera:Hesp eridae) from southern andra[radish,Centre for Info BioTecnology,4(1):45-51

Sundaraju D.1984.Cahew pests and their natural enemies in Goa, Journal of Platntation Crop, 12(1):33-46.

Tripathy M.K, Naik BS and Mishra HP 2016. Performance of emamectin benzoate against major insect pest attacking paddy.Journal of applied Zoological 
Research,27(2):149-152

Tripathy, M. K, Rout M and TripathyA. 2018.

Population dynamics of teak defoliator,
Hyblaeapuera Cramer at Coastal Odisha, India. Journal of Entomology and Zoology studies 6(5) 2378-2387.

\section{How to cite this article:}

Jyoti Kumari, M. K. Tripathy and Harekrushna Das. 2020. Diversity of Insect and Non Insect Pest Infesting Karanja, Pongamia pnnata (L.) Pierre at Bhubaneswar, Odisha, India and their Natural Enemies. Int.J.Curr.Microbiol.App.Sci. 9(07): 1577-1596.

doi: https://doi.org/10.20546/ijcmas.2020.907.183 